\title{
MODELOS DE SIMULAÇÃO PARA CLASSE DIAMÉTRICA EM Populus sp.
}

\section{Simulation Models for Diametric Class in Populus sp.}

\author{
Ana Paula Dalla Corte ${ }^{1}$ \\ Carlos Roberto Sanquetta ${ }^{2}$ \\ Diego Morel Berni ${ }^{3}$
}

\section{Resumo}

A prognose da distribuição diamétrica é muito utilizada para o planejamento florestal, visando a orientar as atividades do manejador a respeito do volume de madeira por classes diamétricas nas diferentes idades do povoamento. O objetivo deste trabalho foi desenvolver um modelo para simular o comportamento da distribuição diamétrica nas diferentes idades de um povoamento de Álamo (Populus sp.). Os dados utilizados neste trabalho são oriundos de povoamentos de Álamo, situados no Estado do Paraná, de diferentes idades. O modelo ajustado para classificar o sítio do povoamento apresentou um coeficiente de determinação de 0,74 e erro padrão da estimativa de 2,19 m (16,11\%). 0 modelo ajustado para predizer a sobrevivência por hectare nas diferentes idades obteve um coeficiente de determinação de 0,88 e um erro padrão da estimativa de 7,47 árv./ha ou 2,59\%, considerado um erro bom.

Palavra-chave: Simulação; Distribuição diamétrica; Álamo.

\section{Abstract}

The prognosis of the diametric distribution is very used for the forest planning seeking to guide the manager's activities in what says about the wood volume for diametric classes in different ages of the stand. The objective of this work was to develop a model to simulate the behavior of the diametric distribution in the different ages of a "Alamo" (Populus sp.) stand. The data used in this work are originating from a Alamo stand of different ages, located in the State of Parana. The adjusted model to classify the site of the stand presented a determination coefficient of 0,74 and a standard error of $2,19 \mathrm{~m}(16,11 \%)$. In order to predict the survival per hectare in the different ages of the stand the adjusted model obtained a determination coefficient of 0,88 and a standard error of 7,47 trees / ha or 2,59\%, that it is considered as a low level error.

Keywords: Prognosis; diametric distribution; Alamo.

1 Engenheira Florestal, Mestranda do Curso de Engenharia Florestal, Professora da Universidade Federal do Paraná, Bolsista do CNPq. e-mail: anacorte@floresta.ufpr.br

2 Engenheiro Florestal, Professor do Departamento de Ciências Florestais, Universidade Federal do Paraná, Bolsista do CNPq. email: sanqueta@floresta.ufpr.br

3 Acadêmico do curso de Engenharia Florestal, Universidade Federal do Paraná, Bolsista PET. e-mail: berni@floresta.ufpr.br 


\section{Introdução}

As empresas florestais têm procurado ao máximo otimizar suas atividades com a finalidade de maximizar seus lucros e minimizar seus custos, mantendo a qualidade (CUNHA NETO, 1994). Assim, todas as ferramentas que possibilitem um melhor planejamento das atividades do manejador são úteis e devem ser consideradas. Neste grupo de ferramentas está a prognose das classes diamétricas, que apóiam o planejamento do volume de madeira por classe diamétricas nos diferentes períodos.

Thiersch (1997) cita que por meio da compreensão da distribuição diamétrica das árvores que compõem um povoamento florestal é possível identificar seu potencial de uso presente ou futuro. Complementa ainda que desenvolver um sistema de predição presente e futura propicia: definir, por sítio, a rotação econômica ótima; simular diferentes opções de desbastes; definir a densidade inicial de plantio, a época e a intensidade de desbaste, dentre outras possibilidades.

Existem diferentes tipos de modelos usados para esta finalidade, dependendo dos dados disponíveis e das informações requeridas. Sanquetta (1996) cita que os três principais modelos não espaciais, disponíveis para a predição por classe do povoamento são: funções probabilísticas, matrizes de transição e processos de difusão. As funções de probabilidade expressam a evolução das classes do povoamento.

Conforme Barra (2003), as distribuições tradicionalmente avaliadas na área florestal são: Beta, Gamma, Log-normal, Normal, $\mathrm{S}_{\mathrm{B}}$ Johnson e Weibull de dois ou de três parâmetros. O mesmo autor acrescenta que Glade (1986) e Scolforo (1998) apresentaram uma extensa relação de pesquisas que avaliaram estas funções na área florestal, com diversos graus de sucesso.

Thiersch (1997) testou a eficiência das distribuições: Normal, Log-normal, Gamma, Beta, $S_{B}$ e Weibull por diferentes métodos de ajustes para Eucalyptus camaldulensis onde conclui que as distribuições Beta e $\mathrm{S}_{\mathrm{B}}$ foram as mais eficientes.

Conforme Silva (1986, citado por Guimarães, 1994) a função Weibull é atualmente a mais utilizada nos trabalhos referentes à ajuste de distribuições diamétricas, em povoamentos florestais equiâneos.
Segundo Bailey (1973, citado por Maestri, 2003) a distribuição de probabilidade Weibull foi proposta primeiramente por Fisher e Tippet em 1928, tendo sido desenvolvida independentemente por Waloddi Weibull, físico sueco, em 1939, no estudo de resistência de materiais. Provavelmente depois da segunda Guerra Mundial, com ênfase no estudo da resistência dos materiais, o trabalho de Weibull se destacou e a distribuição passou a ser chamada pelo seu nome. Na literatura Russa, é reconhecida ainda a importância de outro autor, sendo a distribuição normalmente chamada de “WEIBULL-GNEDENKO” (JOHNSON; KOTZ, 1970). A partir de então, praticamente todos os estudos de distribuição de freqüência a tem utilizado (LEITE, 1990 citado por MAESTRI, 2003).

\section{Materiais e métodos}

Os dados utilizados neste estudo são provenientes da espécie Álamo (Populus spp.) oriundos de plantios localizados no Estado do Paraná. Foram coletados dados de diâmetros e alturas em parcelas permanentes em dois momentos. Ainda, foram retirados discos para realização de análises de tronco (ANATRO) com a finalidade de reconstruir os seus padrões de crescimento. Foram amostradas 105 parcelas, com tamanhos variando entre 380 e $900 \mathrm{~m}^{2}$.

\section{Modelos de sítio}

Para Scolforo (1993, citado por CUNHA NETO, 1994) a determinação da qualidade de sítio pode ser feita por métodos diretos e indiretos. Complementa ainda que a escala desejada e a variabilidade dos fatores de sítio determinam qual dos principais fatores é usado para a subdivisão primária.

Campos (1985) acrescenta que a determinação do índice de sítio é um meio usualmente empregado em manejo florestal para classificar os povoamentos equiâneos segundo sua capacidade produtiva.

Foram ajustados modelos para a classificação do sítio, para sobrevivência, área basal e ajuste da distribuição diamétrica. A idade índice adotada para a análise da produção foi de 10 anos. 
Para a classificação do sítio foram testados três modelos, onde o melhor, segundo o indicador de ajustes, foi selecionado. O ajuste dos modelos foi efetuado usando-se o software Statistica v.5. Os modelos testados estão apresentados abaixo:

Modelo de Chapman-Richards:

$$
\mathrm{H}_{\mathrm{dom}}=\left(\beta_{0}\left(1-\beta_{1} \mathrm{e}^{\left(-\beta_{2} * \text { Idade }\right)}\right) \frac{1}{1-\beta_{3}}\right)
$$

\section{Modelo de Prodan:}

$$
\mathrm{H}_{\mathrm{dom}}=\frac{\text { Idade }^{2}}{\beta_{0}+\beta_{1} * \text { Idade }+\mathrm{B}_{2} * \text { Idade }^{2}}
$$

Modelo de Schumacher:

$$
\mathrm{H}_{\text {dom }}=\beta_{0} \mathrm{e}\left(\frac{-\beta_{1}}{\text { Idade }}\right)
$$

Em que:

$$
\mathrm{H}_{\mathrm{dom}}=\text { Altura dominante; }
$$

$\beta_{0^{\prime}} ; \beta_{1} ; \beta_{2}$ e $\beta_{3}=$ coeficientes a serem ajustados para os modelos.

Com a equação selecionada, traçou-se a curva guia ou curva índice, que servirá de apoio para a construção das curvas de índice de sítio. A equação selecionada foi modificada para que 0 cálculo das curvas limites fosse direto.

Como os dados coletados não apresentaram uma grande dispersão, em relação à idade e às alturas dominantes, optou-se por utilizar apenas três classes de sítios com intervalos de $2,7 \mathrm{~m}$ na idade de 10 anos (idade de referência).

\section{Modelos de sobrevivência}

Segundo Clutter et al. (1983), para a construção de modelos que expressem a sobrevivência ou mortalidade das árvores em um povoamento, é necessário que parcelas permanentes instaladas no povoamento sejam acompanhadas em mais de uma medição.
Maestri (2003) cita que muitos trabalhos evidenciam a influência da capacidade produtiva (sítio) na curva de sobrevivência em plantios. Maestri (1992) afirma que as formulações matemáticas dos modelos para estimativa do número de árvores são normalmente dependentes da idade inicial, do intervalo de tempo e, em alguns casos, de alguma expressão de sítio. Os modelos testados para expressar a sobrevivência das árvores foram:

Modelo de Clutter:

$N_{2}=N_{1}\left(\frac{A_{2}}{A_{1}}\right)^{\beta_{1}} \mathrm{e}\left[\left(\beta_{0}+\beta_{2} S\right) *\left(A_{2}-A_{1}\right)\right]$

Modelo de Silva:

$$
\mathrm{N}_{2}=\mathrm{N}_{1} \mathrm{e}\left[\beta_{0}\left(\beta_{1}^{\mathrm{A}_{2}}-\beta_{1}^{\mathrm{A}_{1}}\right)\right]
$$

Em que:

$\mathrm{A}_{1}=$ Idade inicial;

$\mathrm{A}_{2}=$ Idade final;

$\mathrm{N}_{1}=$ Número de árvores por hectare na idade inicial $\mathrm{A}_{1}$;

$\mathrm{N}_{2}=$ Número de árvores por hectare na idade final $\mathrm{A}_{2}$;

$\mathrm{S}=$ Índice de sítio;

$\mathrm{e}=$ é a base do logaritmo natural;

$\beta_{0} ; \beta_{1} ; \beta_{2}$ e $\beta_{3}=$ coeficientes a serem ajustados para os modelos.

\section{Modelo de área basal}

Clutter (1983) diz que a predição da produção futura dos modelos explícitos recebe a denominação de modelo de projeção, a partir de um ano de referência, e considera-se as mesmas variáveis explicativas nos anos respectivos. Já o modelo de predição incorpora variável do povoamento e a idade de prognose como variáveis explicativas. Os modelos utilizados por Maestri (2003) podem ser considerados como exemplos dessa definição.

Maestri (2003) acrescenta que a formulação proposta por ele em seus estudos permite utilizar uma informação conhecida de um inventário 
na idade inicial (A1) ou idade zero. No primeiro caso seria o modelo de projeção e no segundo o de predição.
O modelo testado para predizer a área basal em diferentes momentos do povoamento foi:

$$
\operatorname{Ln}\left(G_{2}\right)=\beta_{0}+\beta_{1} * S+\beta_{2} * \frac{1}{A_{2}}+\beta_{3} *\left(\frac{A_{1}}{A_{2}}\right) * \operatorname{Ln}\left(\beta_{1}\right)+\beta_{4} *\left(1-\frac{A_{1}}{A_{2}}\right)+\beta_{5} * S *\left(1-\frac{A_{1}}{A_{2}}\right)
$$

Em que:

$\mathrm{G}_{1}=$ Área basal por hectare na idade final;

$\mathrm{S}=$ Índice de sítio;
$\mathrm{A}_{1}=$ Idade inicial;

$\mathrm{A}_{2}=$ Idade final;

$\beta_{0} ; \beta_{1} ; \beta_{2} ; \beta_{3} ; \beta_{4}$ e $\beta_{5}=$ coeficientes $\mathrm{a}$ serem ajustados para os modelos.

\section{Modelo para as distribuições diamétricas}

Para as florestas plantadas, é importante que se tenha conhecimento das distribuições diamétricas, para que as funções de prognose da produção possam ser construídas. Muitos autores citam a função de Weibull de 2 ou de 3 parâmetros como sendo uma função bastante flexível, podendo ajustar-se tanto a dados de florestas nativas (decrescente) como a dados de florestas plantadas equiâneas com distribuição unimodal.

A distribuição selecionada para representar a densidade por classe diamétrica do povoamento foi a de Weibull 3P. Esta função é citada por Maestri (2003) por poder assumir diversas formas e ter grande aplicação da distribuição diamétrica das árvores de um povoamento florestal. Conforme esse autor, inúmeros trabalhos de modelagem da estrutura dos povoamentos florestais, utilizando essa função probabilística são encontrados na literatura florestal brasileira e mundial, entre eles Bailey e Dell (1973), Cao etal. (1982), Clutter etal. (1983), Finger (1979), Couto (1980), Burk e Newberry (1984), Glade (1986), Silva (1987), Scolforo (1990), Maestri (1992) e Maestri (1995).

De acordo com Maestri (2003), a função Weibull 3P é descrita pela seguinte função:

$$
f(\mathrm{x})=\left(\frac{\mathrm{c}}{\mathrm{b}}\right) *\left(\frac{\mathrm{DAP}-\mathrm{a}}{\mathrm{b}}\right)^{\mathrm{c}-1} \mathrm{e}^{-\left(\frac{\mathrm{DAP}-\mathrm{a}}{\mathrm{c}}\right)^{\mathrm{c}}}
$$

Maestri (2003) complementa que os coeficientes "a", "b" e "c" são responsáveis pela locação, escala e forma, respectivamente, e seus valores podem ser recuperados, através de diversos métodos, a partir das informações do povoamento florestal. A técnica dos momentos, segundo Scolforo (1998), estabelece que o coeficiente de variação em diâmetro tem relação com o parâmetro da forma da distribuição pela seguinte igualdade:

$\mathrm{CV}_{\mathrm{DAP}}=\frac{\sigma}{\overline{\mathrm{d}}}=\frac{\sqrt{\Gamma\left(1+\frac{2}{\mathrm{c}}\right)-\Gamma^{2}}\left(1+\frac{1}{\mathrm{c}}\right)}{\Gamma\left(1+\frac{1}{\mathrm{c}}\right)}$

Em que:

$\mathrm{CV}_{\mathrm{DAP}}=$ Coeficiente de variação em diâmetro;

$\sigma=$ Desvio padrão do diâmetro ou raiz quadrada da variância do diâmetro;

$\overline{\mathrm{d}}=$ Diâmetro médio aritmético do povoamento;

$\Gamma=$ Função gama;

c = Parâmetro responsável pela forma da função probabilística de Weibull.

Maestri (2003) complementa que pelo processo iterativo, o parâmetro " $\mathrm{C}$ " é recuperado quando a igualdade for estabelecida. Uma vez recuperado o parâmetro da forma, então o parâmetro de escala "b" é recuperado por:

$$
\mathrm{b}=\frac{\overline{\mathrm{d}}}{\Gamma\left(1+\frac{\mathrm{l}}{\mathrm{c}}\right)}
$$


Em que:

$\mathrm{b}=$ Parâmetro responsável pela escala da função probabilística de Weibull;

$\overline{\mathrm{d}}=$ Diâmetro médio aritmético do povoamento;

$\Gamma$ = Função gama;

c = Parâmetro de forma já definido pela equação.

Segundo Maestri (2003), o parâmetro "a" pode ser obtido independentemente. Com isto, seu valor é determinado dentro do processo de compatibilização da área basal advinda da distribuição (soma das áreas transversais de cada classe diamétrica) com a área basal estimada pelo modelo global (EQUAÇÃO 6). Como o parâmetro "a” é responsável pela locação (início da distribuição), então uma variação em sua magnitude irá "arrastar" a distribuição para um dos lados, levando a diferentes valores de área basal, originada da função de distribuição de diâmetros. Assim, também por processo iterativo, obtémse 0 valor do parâmetro de locação quando alcançada tal igualdade, ou seja, para diferentes valores de "a", estima-se a distribuição diamétrica pela função Weibull (parâmetros "c" e "b" já recuperados pelas formulações (equação 8) e (equação 9) e calcula-se a área basal resultante pela soma das áreas transversais de todas as classes de diâmetro. $\mathrm{O}$ valor do parâmetro "a" que estabelece a similaridade (menos de $0,1 \%$ de diferença) entre a área basal do modelo global (equação 6) com aquela obtida pelo processo iterativo, será considerado como ideal.

A predição volumétrica para a distribuição diamétrica foi realizada utilizando-se o modelo 10:

$$
\begin{aligned}
\mathrm{v}=\beta_{0}+\beta_{1} * \mathrm{DAP}+\beta_{2} * \mathrm{DAP} * \mathrm{H}+\beta_{3} * \mathrm{DAP}^{2} * \mathrm{H}(10) \\
\mathrm{v}=\text { Volume }(\mathrm{m} 3) ; \\
\text { DAP = Diâmetro a altura do peito; } \\
\mathrm{H}=\text { Altura total; } \\
\beta_{0} ; \beta_{1} ; \beta_{2} \text { e } \beta_{3}=\text { coeficientes a serem ajus- }
\end{aligned}
$$

tados para os modelos.

\section{Resultados e discussões}

Índice de sítio

Abaixo está apresentada a equação ajustada e selecionada para expressar o sítio no povoamento em questão, ou seja, o modelo de Chap-
man-Richards (EQUAÇÃO 11). Considera-se que o índice de sítio $(S)$ é igual à altura dominante quando a idade (A) for igual à idade índice (Ai) e que esta idade é de 10 anos, então o valor índice de sítio é de 16,00 metros. Na tabela 1, apresentada abaixo, estão descritas as estatísticas resultantes do ajuste do modelo selecionado.

$$
\mathrm{H}_{\text {dom }}=\left(18,29514\left(1-0,03997 \mathrm{e}^{(-0,303350 \times \text { dade })}\right) \frac{1}{1-0,979293}\right)
$$

\section{Tabela 1 - Principais estatísticas resultantes do ajuste do modelo de Chapman-Richards.}

Table 1 - Main statistics resulted from the adjustment of the Chapman-Richards's model.

\begin{tabular}{ll}
\hline Estatísticas & \\
\hline Número de observações & 1028 \\
$\mathrm{R}^{2}{ }_{\text {ajustado }}$ & 0,74 \\
Erro padrão da estimativa recalculado (m) & 2,19 \\
Erro padrão da estimativa recalculado (\%) & 16,11
\end{tabular}

Como os valores observados para a altura dominante, aos 10 anos de idade, variaram entre 12,5 a $21 \mathrm{~m}$, optou-se por estabelecer 3 classes, com amplitude de 2,7m, sendo elas: 12,0, 14,7 e 17,3m (TABELA 2).

\section{Tabela 2 - Valores de altura dominante para os três índices de sítio.}

Table 2 - Dominant high values for three site index.

\begin{tabular}{lrrrrrr}
\hline \multirow{2}{*}{ Idade } & \multicolumn{6}{c}{ Classe III -IS = 13,3m Classe II - IS = 16,0m Classe I - IS = 18,7m } \\
\cline { 2 - 7 } & $\mathbf{U}(\mathbf{1 2 , 0 )}$ & $\mathbf{L S}(\mathbf{1 4}, \mathbf{7})$ & $\mathbf{L I}(\mathbf{1 4 , 7 )}$ & $\mathbf{L S}(\mathbf{1 7 , 5 )}$ & $\mathbf{U}(\mathbf{1 7 , 5 )}$ & $\mathbf{L S ( 2 0 , 0 )}$ \\
\hline 1 & 3,10 & 3,79 & 3,79 & 4,48 & 4,48 & 5,17 \\
2 & 4,54 & 5,55 & 5,55 & 6,56 & 6,56 & 7,57 \\
3 & 6,02 & 7,35 & 7,35 & 8,69 & 8,69 & 10,03 \\
4 & 7,40 & 9,04 & 9,04 & 10,68 & 10,68 & 12,33 \\
5 & 8,61 & 10,52 & 10,52 & 12,43 & 12,43 & 14,34 \\
6 & 9,62 & 11,76 & 11,76 & 13,90 & 13,90 & 16,04 \\
7 & 10,45 & 12,77 & 12,77 & 15,09 & 15,09 & 17,41 \\
8 & 11,10 & 13,57 & 13,57 & 16,04 & 16,04 & 18,50 \\
9 & 11,61 & 14,19 & 14,19 & 16,77 & 16,77 & 19,35 \\
10 & 12,00 & 14,67 & 14,67 & 17,33 & 17,33 & 20,00 \\
11 & 12,30 & 15,03 & 15,03 & 17,76 & 17,76 & 20,49 \\
12 & 12,52 & 15,30 & 15,30 & 18,08 & 18,08 & 20,86 \\
\hline
\end{tabular}


Na Figura 1 estão apresentadas as Classes Índices de Sítio, correspondendo à altura dominante das classes na idade de referência.

\section{Figura 1 - Curvas de Índice de Sítio para 0 povoamento de Álamo.}

Figure 1 -Site index curves for the "Álamo" stand.



\section{Sobrevivência}

O modelo selecionado para predizer a sobrevivência das árvores do povoamento foi 0 de Clutter (12).

$N_{2}=N_{1}\left(\frac{A_{2}}{A_{1}}\right)^{-0.010505} \mathrm{c}\left[(-0,021352+0,000423 * S) *\left(A_{2}-A_{1}\right)\right](12)$

O modelo mencionado apresentou um coeficiente de determinação de $88 \%$ e um erro padrão da estimativa de 7,47 árvores por hectare, ou 2,59\%, considerado bom no âmbito florestal (TABELA 3).

\section{Tabela 3 - Principais estatísticas} resultantes do ajuste do modelo de Clutter. Table 3 - Main statistics resulted from the adjustment of the Clutter model.

\begin{tabular}{ll}
\hline Estatísticas & \\
\hline Número de observações & 169 \\
$\mathrm{R}^{2}{ }_{\text {ajustado }}$ & 0,88 \\
Erro padrão da estimativa recalculado (indiv.) & 7,47 \\
Erro padrão da estimativa recalculado (\%) & 2,59 \\
\hline
\end{tabular}

Outra análise realizada para verificar 0 comportamento do modelo selecionado foi a verificação da função lógica do modelo. Ou seja, que o modelo em questão seja realmente compatível. Essa propriedade é citada por Clutter et al. (1983) e Sanquetta (1996). O resultado deste teste está apresentado na figura 2 .

\section{Figura 2 - Teste lógico do modelo selecionado para as estimativas do número de árvores.}

Figure 2 - Logical test of the selected model for tree number estimates

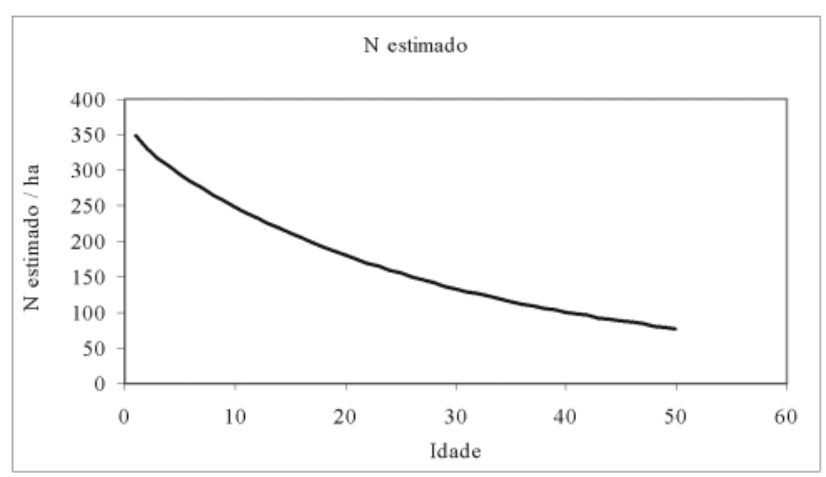

Esta propriedade é explicada como tendo que atender aos seguintes itens:

- Se $A_{t}$ é igual a $A_{0}, N_{t}$ deve tender a $\mathrm{N}_{0}$;

- Para povoamentos equiâneos quando $\mathrm{A}_{\mathrm{t}} \rightarrow \infty$ então $\mathrm{N}_{\mathrm{t}} \rightarrow 0$;

- Se a função é usada para predizer $\mathrm{Nt}$ na idade $A_{t}$ e $N_{t^{\prime}}$ e $N_{t+1}$ na idade $A_{t+1}\left(A_{t+1}>A_{t}>A 0>\right)$ 0 resultado deve ser igual à projeção direta de $A_{0}$ até $\mathrm{A}_{\mathrm{t}+1}$;

Área basal

O modelo ajustado para as estimativas de área basal selecionado está apresentado abaixo (13). Os coeficientes ajustados foram significativos a ao nível de $5 \%$.

$$
\begin{aligned}
& \operatorname{Ln}\left(G_{2}\right)=0.0503+0.1035 * S-1.1957 * \frac{1}{A_{2}}+ \\
& 0.4248 *\left(\frac{A_{1}}{A_{2}}\right) * \operatorname{Ln}(0.1035)-1.1957 *\left(1-\frac{A_{1}}{A_{2}}\right)+0.4054 * S *\left(1-\frac{A_{1}}{A_{2}}\right)
\end{aligned}
$$

Efetuou-se uma análise da área basal estimada pelo modelo global e da área basal observada. Posteriormente efetuou-se o ajuste do modelo para predição das distribuições diamétricas (Weibull 3P). O valor do padrão de forma (c) foi 
encontrado utilizando-se os coeficientes de variação dos diâmetros (TABELA 4).

\section{Tabela 4 - Coeficiente de Variação do} diâmetro e parâmetros encontrados para a Função Weibull 3P.

Table 4 - Coefficient of variation for diameter estimates and the three parameters of the Weibull function.

\begin{tabular}{lllll}
\hline Idade & $\mathbf{C V}$ & $\mathbf{a}$ & $\mathbf{b}$ & $\mathbf{C}$ \\
\hline 1 & 0,27 & 2,15 & 2,37 & 4,17 \\
2 & 0,36 & 2,08 & 3,98 & 3,02 \\
3 & 0,38 & 3,59 & 5,62 & 2,85 \\
4 & 0,40 & 3,00 & 7,30 & 2,69 \\
5 & 0,37 & 3,00 & 9,22 & 2,94 \\
6 & 0,33 & 3,00 & 11,10 & 3,34 \\
7 & 0,30 & 3,00 & 12,61 & 3,71 \\
8 & 0,25 & 3,00 & 14,01 & 4,53 \\
\hline
\end{tabular}

As projeções diamétricas para o número de árvores simuladas até a idade de 8 anos estão apresentadas na figura 3.

\section{Figura 3 - Evolução das distribuições diamétricas nas diferentes idades pela função Weibull 3P.}

Figure 3 - Evolution of the diameter distribution in different ages, using the Weibull 3P function.

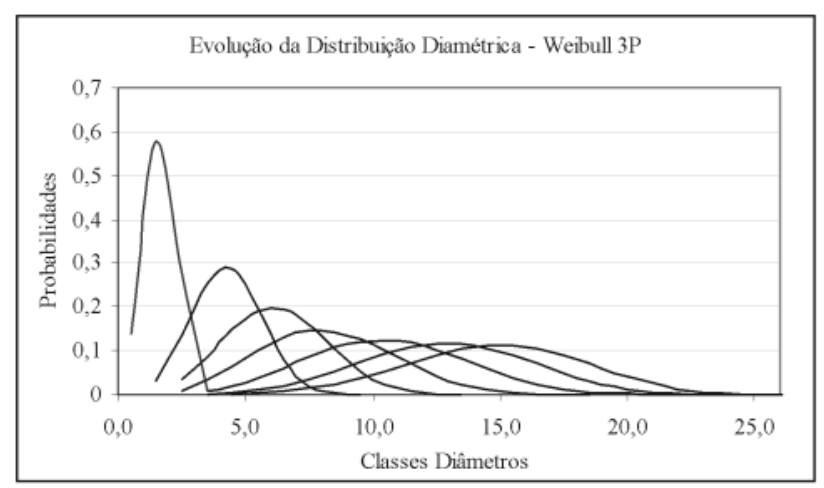

Volumes

Na Figura 4 está apresentada a simulação do comportamento temporal dos volumes em função das distribuições diamétricas estimadas pela função Weibull 3P. Para se chegar em tais resultados, estimaram-se os volumes com a função já ajustada pela empresa para o povoamento e, posteriormente, multiplicaram-se as projeções para as classes diamétricas pelo volume estimado pela idade.

\section{Figura 4 - Evolução dos volumes nas dife- rentes idades em função da predição da distribuição diamétrica.}

Figure 4 - Volume evolution in different ages as a function of the diameter distribution prediction.

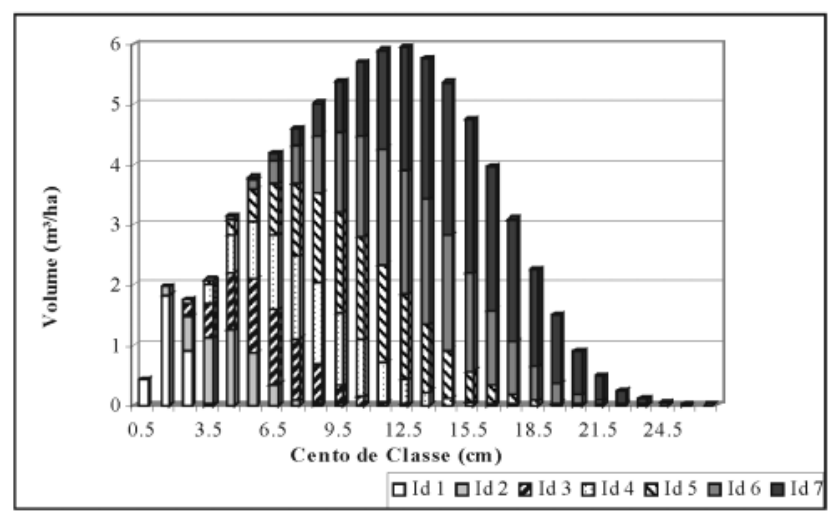

\section{Conclusões}

Pode-se concluir que esta metodologia, utilizada para a recuperação dos parâmetros da função Weibull 3P conseguiu representar o povoamento em questão. Porém, devido à falta de dados para indivíduos com idades superiores a 8 anos, não foi possível predizer a partir desta idade. Ainda, deve-se ressalvar que a aplicação deste modelo deve se restringir a povoamentos de Álamo com semelhantes condições de produção aos utilizados para o presente trabalho.

Ainda poder-se-ia utilizar esta situação para a projeção dos volumes por classes diamétricas, bastando para tanto gerar estimativas de volume e combiná-las com os números de árvores obtidos por simulação nas respectivas classes. 


\section{Referências}

BARRA, O. S. V. Sistema de simulação de crescimento e produção de Populus spp. no Chile (Sistema Salica). 2003. 127f. Tese (Doutorado em Engenharia Florestal) - UFPR. Curitiba, 2003.

CAMPOS, J. C. C.; TORQUATO, M. C.; RIBEIRO, G. A. Equações para calcular índices de local e incremento da altura em plantações puras de Eucalyptus grandis. Revista Árvore, Viçosa, v. 9, n. 1, p. 1-9, 1985.

CLUTTER, J. L et al. Timber management: a quatitative approach. New York, John Willey e Sons, p. 333, 1983.

CUNHA NETO, F. R. da Sistema para predição presente e futura da produção por classe de diâmetro utilizando a função Weibull, para Eucalyptus grandis e Eucalyptus urophylla. 1994. 159 f. Dissertação (Mestrado) - Universidade Federal de Lavras. Dissertação apresentada a Universidade Federal de Lavras, Lavras, 1994.

GUIMARÃES, D. F. Desenvolvimento de um modelo de distribuição diamétrica de passo invariante para prognose e projeção da estrutura de povoamentos de Eucalipto. 1994. $160 \mathrm{f}$. Tese (Doutorado em Engenharia Florestal) - Universidade Federal de Viçosa, 1994.
MAESTRI, R. Modelo de Crescimento e produção para povoamentos clonais de Eucalyptus grandis considerando Variáveis Ambientais. 2003, 143 f. Tese (Doutorado em Engenharia Florestal) - UFPR. Curitiba, 2003.

SANQUETTA, C. R. Fundamentos biométricos dos modelos de simulação florestal. Curitiba: Fundação de Pesquisas Florestais do Paraná, 1996. p. 49. (Série didática, n. 08).

SCOLFORO, José Roberto Soares. Modelagem do crescimento e da produção de florestas plantadas e nativas. LAVRAS: UFLA/ FAEPE, 1998. v. 1. p. 443.

THIERSCH, A. Eficiência das distribuições diamétricas para prognose da produção de Eucalyptus camaldulensis. 1997. 155 f. Tese (Doutorado em Engenharia Florestal) - Lavras, 1997. 